\title{
Lack of evidence for interventions offered in UK fertility centres
}

\author{
Carl Heneghan and colleagues call for better quality evidence to help people seeking assisted \\ reproduction make informed choices
}

Carl Heneghan professor, E A Spencer researcher, N Bobrovitz researcher, D R J Collins researcher, D Nunan researcher, A Plüddemann researcher, OA Gbinigie researcher, I Onakpoya researcher, J O'Sullivan researcher, A Rollinson research assistant, A Tompson researcher, B Goldacre consultant, K R Mahtani general practitioner

Centre for Evidence-Based Medicine, Nuffield Department Primary Care Health Sciences, University of Oxford, Oxford, UK

Infertility affects about one in seven couples, many of whom seek medical help to have a child. ${ }^{1}$ Although numerous new fertility interventions and products have been developed over the past decade, there are concerns that some might not be evidence based and that some clinics may be offering additional services that are not based on the most up-to-date research. ${ }^{2}$

In vitro fertilisation (IVF) is expensive-a single cycle can cost $£ 5000$ ( $€ 5800 ; \$ 6200$ ) — and places considerable financial burden on patients, especially as $59 \%$ of procedures are not NHS funded. ${ }^{3}$ On top of this UK fertility treatment centres offer a range of additional investigations and treatments, ${ }^{4}$ costing from $£ 50$ for a single screening blood test to as much as $£ 8000$ for egg freezing packages (box 1).

Guidance from the UK regulator, the Human Fertilisation and Embryology Authority (HFEA), suggests questions that couples might want to ask before deciding on treatment. ${ }^{2}$ These include: Is this treatment recommended by the National Institute for Health and Care Excellence (NICE) and, if not, why not? Has this treatment been subjected to randomised controlled clinical trials that show it is effective and is there a Cochrane review available? Are there any adverse effects or risks (known or potential) of the treatment? ${ }^{2}$ Given concerns over the evidence base for fertility treatments and the implications for patients we set out to answer these questions.

\section{What did we do?}

We obtained from HFEA a list of all UK centres providing fertility treatments and examined their websites. From these sites we compiled a list of interventions offered in addition to standard IVF that are claimed to improve fertility outcomes. ${ }^{4}$ We excluded interventions aimed at patients with a pre-existing disease such as diabetes, diagnosed conditions such as polycystic ovarian syndrome, or neurological conditions such as spinal cord injury; we excluded interventions related to donation of sperm or eggs; and we excluded complementary therapies such as homeopathy and nutrition. This gave a list of 38 fertility interventions. We classified 27 of these as add-on interventions, six as alternatives to IVF, and five as treatments for preservation of fertility (box 2).

For these 38 additional fertility interventions, we searched for evidence as HFEA suggests, focusing on the key outcome of live birth rates (see table A on thebmj.com). Nine researchers (NB, DC, OG, KM, DN, IO, AP, ES, JOS) independently searched Medline and the Cochrane library for systematic reviews and randomised controlled trials in April 2016. When we could not find any randomised controlled trials, we looked for the next highest level of available evidence (eg, observational study) and categorised the evidence found using the Oxford Centre for Evidence Based Medicine levels of evidence. ${ }^{5} \mathrm{We}$ also searched the Cochrane reviews and NICE guidance CG156 for information on harms up to September 2016.

\section{What did we find?}

\section{National Institute for Health and Care Excellence recommendation}

NICE provides clear advice for 13 (34\%) of the 38 interventions investigated. Of these, 11 are recommended only in specific populations-for example, measurement of thyroid function should be offered only to women with symptoms of thyroid disease - and two are not recommended (hysteroscopy, assisted hatching). There was no clear guidance for 19 interventions, and six interventions were recommended for research only (figure $\Downarrow$ ). 


\section{Box 1: Example of costs for interventions additional to standard IVF}

Individual screening blood tests-start at £50

Embryoglue-up to $£ 160$

Intralipid infusions-up to $£ 250$

Endometrial scratch—up to $£ 325$

Assisted hatching - up to $£ 450$

Blastocyst culture-up to $£ 800$

Time lapse imaging - up to $£ 850$ for the Eeva time lapse incubator, up to $£ 800$ for the Embryoscope

Intracytoplasmic morphological sperm injection (IMSI)—up to £1855

Percutaneous epididymal sperm aspiration/testicular sperm extraction (PESE/ TESE)—up to $£ 1600$

Preimplantation genetic screening $-£ 3500$

Egg freezing packages-up to $£ 8000$

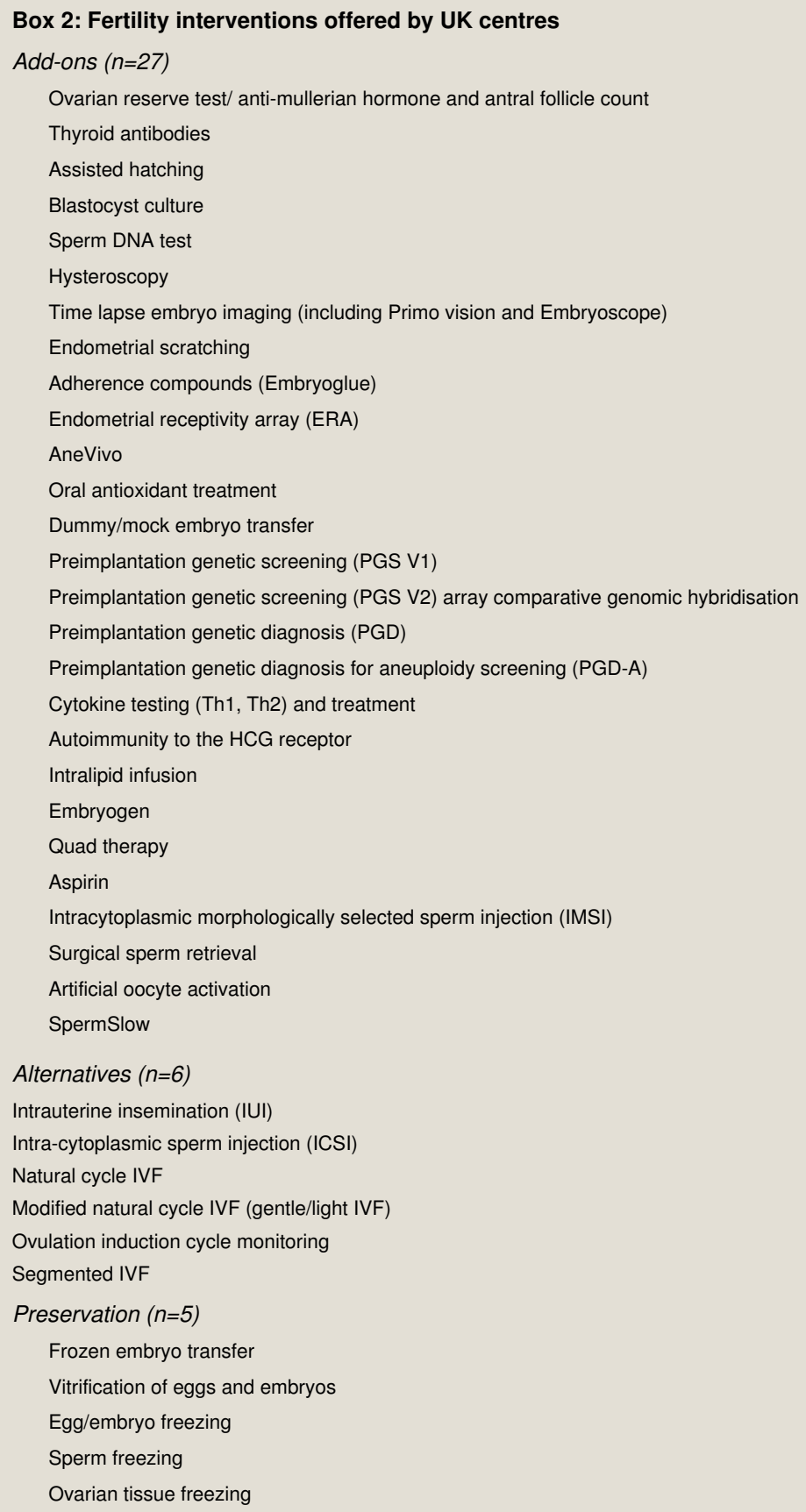

\section{Box 2: Fertility interventions offered by UK centres}

Add-ons ( $n=27$ )

Ovarian reserve test/ anti-mullerian hormone and antral follicle count

Thyroid antibodies

Assisted hatching

Blastocyst culture

Sperm DNA test

Hysteroscopy

Time lapse embryo imaging (including Primo vision and Embryoscope)

Endometrial scratching

Adherence compounds (Embryoglue)

Endometrial receptivity array (ERA)

AneVivo

Oral antioxidant treatment

Dummy/mock embryo transfer

Preimplantation genetic screening (PGS V1)

Preimplantation genetic screening (PGS V2) array comparative genomic hybridisation

Preimplantation genetic diagnosis (PGD)

Preimplantation genetic diagnosis for aneuploidy screening (PGD-A)

Cytokine testing (Th1, Th2) and treatment

Autoimmunity to the HCG receptor

Intralipid infusion

Embryogen

Quad therapy

Aspirin

Intracytoplasmic morphologically selected sperm injection (IMSI)

Surgical sperm retrieval

Artificial oocyte activation

SpermSlow

Alternatives ( $n=6)$

Intrauterine insemination (IUI)

Intra-cytoplasmic sperm injection (ICSI)

Natural cycle IVF

Modified natural cycle IVF (gentle/light IVF)

Ovulation induction cycle monitoring

Segmented IVF

Preservation ( $n=5)$

Frozen embryo transfer

Vitrification of eggs and embryos

Egg/embryo freezing

Sperm freezing

Ovarian tissue freezing

Randomised controlled clinical trials and Cochrane reviews
For 27 of the 38 fertility interventions ( $71 \%$ ) we found a relevant systematic review; 18 of these were Cochrane reviews (figure $\downarrow$ ). We also found one Cochrane overview, published in 2015, 
including 59 systematic reviews of the effectiveness of assisted reproductive technologies. ${ }^{6}$ These reviews reported that five of the 38 interventions improved live birth outcomes; for 13 interventions, the evidence was insufficient to make a summary estimate, and for seven there was evidence that the intervention did not improve live birth rates. For preimplantation genetic screening older methods worsened outcomes, but there was some evidence of benefit for more recently developed techniques.

The five interventions for which we found evidence of improvements in live birth rates were blastocyst culture, endometrial scratching, adherence compounds, oral antioxidants, and intrauterine insemination in a natural cycle. However, for all of these interventions, the supporting studies had methodological problems that raise uncertainty about the results (table $\Downarrow$ ).

We also identified one review that showed a negative effect. A 2006 Cochrane review of nine trials of preimplantation genetic screening concluded that it significantly lowered live birth rate, ${ }^{12}$ and a 2009 review also found no benefit. ${ }^{13}$ Newer techniques such as array comparative genomic hybridisation have subsequently been introduced. We identified a 2015 systematic review not done by the Cochrane group assessing four randomised trials of the newer techniques, targeted generally at younger women. ${ }^{14}$ In one trial delivery rates per cycle were higher in the intervention group than the control group but in the pooled analysis including cohort studies, there was no significant effect. NHS England's 2013 clinical commissioning policy reports that in the "absence of evidence of its clinical and cost effectiveness, there is no intention to support the introduction of preimplantation genetic screening into NHS clinical practice." 15

For 11 interventions we were unable to find systematic review evidence (figure $\Downarrow$ ). For eight of these we identified only a single randomised trial or observational study, none of which showed benefit. For three interventions (segmented IVF, dummy embryo transfer, and quad therapy) we were unable to find any evidence beyond expert opinion or mechanism of action.

\section{Known or potential adverse effects or risks}

NICE guidance was not helpful about harms, providing comment for only two interventions (intracytoplasmic sperm injection (ICSI) and ovulation induction and cycle monitoring). For IVF with or without ICSI, NICE guidance states that women should be informed that the absolute risks of long term adverse outcomes are low but that a small increased risk of ovarian tumours cannot be excluded (table B on bmj.com). For ovulation induction and cycle monitoring the guidance states the lowest effective dose and duration of use of ovulation induction or ovarian stimulation agents should be used — an indirect comment on harms.

The Cochrane reviews gave limited information on harms. In many cases the included trials provided no information, inadequate information, or inconsistently reported information on adverse events. For example, the review on assisted hatching reported that miscarriage rates were similar in both groups and multiple pregnancy rates were significantly increased in the assisted hatching group. However, there were insufficient data on ectopic pregnancy, congenital or chromosomal abnormalities, blastocyst formation, or embryo damage. ${ }^{16}$

\section{How robust is the evidence?}

Of the 38 interventions we reviewed, there are only 11 for which NICE guidance recommends use in targeted populations. Our appraisal of the evidence shows only one intervention, endometrial scratching, for which the review evidence robustly supports an increase in live birth rate, yet even this evidence is of only moderate quality, and the observed benefit is only in women with more than two previous embryo transfers. A UK multicentre trial is investigating the use of endometrial injury in women undergoing IVF for the first time, which should provide relevant information for a broader patient population. ${ }^{17}$

Cochrane systematic reviews cover less than half of the available treatments. To find information we had to look at other reviews or lower quality evidence, some of which is not freely available to the public. The reporting of harms was poor and therefore for many treatments it is impossible to be informed about adverse effects.

There is an urgent need for randomised controlled trials for many interventions that are currently being offered. Treatments with uncertain effects (benefits and harms) should be licensed for use only in the context of meaningful research. The benefits and harms of simultaneous use of multiple interventions are also not known.

\section{Providing better evidence}

People seeking fertility treatment need good quality evidence to make informed choices. The current approach by HFEA leaves patients and clinicians to seek evidence for themselves or from staff in private clinics selling fertility services. We do not believe this approach is realistic. Patients may be desperate, and therefore vulnerable, and we have shown that fertility interventions are offered without supporting evidence to back up claims of effect. ${ }^{4}$

Patients are unlikely to have specialist skills in seeking and critically appraising clinical evidence. For complex issues such as genetic screening we consider the HFEA's advice, that "you should talk to your GP to go through the options available," unreasonable, as there is likely to be insufficient knowledge available to correctly reflect the potential benefits and harms of such interventions. ${ }^{2}$

Furthermore, there are no maintained relevant information resources appropriate for use by non-clinicians. Initiatives such as PubMed Health and Cochrane's plain language summaries are welcome but they need to cover all the treatments being offered, be updated regularly, and go beyond published papers to overcome the reporting bias that affects much of the published research in the fertility field.

NICE together with HFEA should provide guidance on what is offered, and a recommendation for or against each intervention. The framework setting out which treatments should be offered in which populations should be clearer to reduce the potential for indication creep, whereby use is expanded from people who have clearly benefited to those for whom the evidence is much more shaky or non-existent. NICE should also consider adopting the GRADE system for making recommendations, which allows for strong (offer to everyone) or weak (offer to certain individuals) recommendations, with or without certain conditions. ${ }^{18}$ Furthermore, recommendations should be based on live birth rates rather than pregnancy rates, which are inadequate for several reasons, not least because about $5 \%$ of pregnancies are lost between the first trimester and birth. ${ }^{19}$

Guidance also needs continually updating if it is to be relevant and meaningful, particularly because of the growing number of 
available tests, techniques, and treatments. Furthermore, clinics' awareness and uptake of guidance needs improving: a recent survey of 46 UK clinics licensed to provide intrauterine insemination found that although most clinics were aware of NICE guidance, only 10 clinics reduced the number of cycles or restricted its use according to NICE recommendations. ${ }^{20}$

Currently, clinics vary widely in what they offer and there is a lack of clarity on what constitutes an add-on intervention. Clear classification systems and better information will aid understanding and ensure couples enter treatment fully informed.

Contributors and sources: This work arose after BBC Panorama asked the CEBM to carry out an independent review of the evidence for fertility treatments additional to IVF. The BBC had no role in the review's protocol, methodology, or interpretation of findings but was kept aware of its progress. Deborah Cohen, the reporter of a BBC Panorama on fertility treatments, is a freelance editor at The $B M J$. $\mathrm{CH}$ devised the study and all authors reviewed the methods. $K M$ and $A R$ managed the data extraction. AT, NB, DN, DC, ES, OG, IO, and AP contributed to the searches for evidence and the data extraction. $\mathrm{CH}, \mathrm{ES}, \mathrm{BG}$, and $\mathrm{KM}$ discussed and analysed the issues arising and all authors commented on and approved the final draft.

Competing interests: We have read and understood BMJ policy on declaration of interests and declare the following: $\mathrm{CH}$ has received grant funding from WHO, the National Institute for Health Research (NIHR), and the NIHR School of Primary Care and occasionally receives expenses for teaching evidence based medicine. He jointly runs the EvidenceLive Conference with BMJ. BG has received research funding from the Laura and John Arnold Foundation, the Wellcome Trust, the $\mathrm{NIHR}$, the Health Foundation, and WHO; he also receives personal income from speaking and writing for lay audiences on the misuse of science. KM has received funding from the NIHR and the RCGP for independent research projects. The views expressed here are those of the authors and not necessarily those of any of the affiliated institutions mentioned in the manuscript.

Provenance and peer review: Not commissioned by The BMJ; externally peer reviewed.

1 NICE. Fertility problems: assessment and treatment. Clinical guideline CG156. https:// www.nice.org.uk/Guidance/CG156

2 Human Fertilisation and Embryology Authority. Getting started: your guide to fertility treatment. http://www.hfea.gov.uk/docs/GSG_amended_September_2016_full_version. pdf
3 Human Fertilisation and Embryology Authority. Fertility treatment 2014: trends and figures. http://www.hfea.gov.uk/docs/HFEA Fertility treatment Trends and figures 2014.pdf a 4Spencer EA, Mahtani KR, Goldacre B, et alClaims for fertility interventions: a systematic assessment of statements on UK fertility centre websites. BMJ Open 2016;6:e13940. doi:10.1136/bmjopen-2016-013940.

5 Howick J, Chalmers I, Glasziou P, et al; OCEBM Levels of Evidence Working Group. The Oxford 2011 levels of evidence. 2012. http://www.cebm.netocebm-levels-of-evidence

6 Farquhar C, Rishworth JR, Brown J, Nelen WL, Marjoribanks J. Assisted reproductive technology: an overview of Cochrane Reviews. Cochrane Database Syst Rev 2015;7:CD010537. doi:10.1002/14651858.CD010537.pub4. pmid:26174592.

7 Glujovsky $D$, et alCleavage stage versus blastocyst stage embryo transfer in assisted reproductive technology. Cochrane Database Syst Rev 2016;6:CD002118. doi:10.1002/ 14651858.CD002118.pub5.

8 Nastri CO, Lensen SF, Gibreel A, et al. Endometrial injury in women undergoing assisted reproductive techniques. Cochrane Database Syst Rev 2015;3:CD009517. doi:10.1002 14651858.CD009517.pub3. pmid:25803542.

9 Bontekoe S, Heineman MJ, Johnson N, Blake D. Adherence compounds in embryo transfer media for assisted reproductive technologies. Cochrane Database Syst Rev 2014;6:CD007421. doi:10.1002/14651858.CD007421.pub3. pmid:24567053.

10 Showell MG, Mackenzie-Proctor R, Brown J, Yazdani A, Stankiewicz MT, Hart RJ. Antioxidants for male subfertility. Cochrane Database Syst Rev 2014;2:CD007411. doi: 10.1002/14651858.CD007411.pub3. pmid:25504418.

11 van Rumste MM, Evers JL, Farquhar CM. Intra-cytoplasmic sperm injection versus conventional techniques for oocyte insemination during in vitro fertilisation in patients with non-male subfertility. Cochrane Database Syst Rev 2003;2:CD001301.pmid:12804403.

12 Twisk M, Mastenbroek S, van Wely M, Heineman MJ, Van der Veen F, Repping S. Preimplantation genetic screening for abnormal number of chromosomes (aneuploidies) in in vitro fertilisation or intracytoplasmic sperm injection. Cochrane Database Syst Rev 2006;1:CD005291.pmid:16437524

13 Checa MA, Alonso-Coello P, Solà I, Robles A, Carreras R, Balasch J. IVF/ICSI with or without preimplantation genetic screening for aneuploidy in couples without genetic disorders: a systematic review and meta-analysis. J Assist Reprod Genet 2009;26:273-83. doi:10.1007/s10815-009-9328-4pmid:19629673.

14 Chen M, Wei S, Hu J, Quan S. Can comprehensive chromosome screening technology improve IVF/ICSI outcomes? A meta-analysis. PLoS One 2015;10:e0140779. doi:10. 1371/journal.pone.0140779pmid:26470028

15 NHS Commissioning Board. Clinical commissioning policy: pre-implantation genetic diagnosis. 2013. https://www.england.nhs.uk/wp-content/uploads/2013/04/e01-p-a.pdf

16 Carney SK, Das S, Blake D, Farquhar C, Seif MM, Nelson L. Assisted hatching on assisted conception (in vitro fertilisation (IVF) and intracytoplasmic sperm injection (ICSI) Cochrane Database Syst Rev 2012;12:CD001894. doi:10.1002/14651858.CD001894.pub5. pmid: 23235584.

17 Endometrial scratch trial. http://www.isrctn.com/ISRCTN23800982?q=\& filters=conditionCategory:Pregnancy\%20and\%20Childbirth\&sort=\&offset=8\& totalResults $=656 \&$ page $=1$ \&pageSize $=10 \&$ searchType=basic-search

18 Guyatt GH, Oxman AD, Vist GE, et al. GRADE Working Group. GRADE: an emerging consensus on rating quality of evidence and strength of recommendations. $B M J$ 2008;336:924-6. doi:10.1136/bmj.39489.470347.ADpmid:18436948.

19 Clarke JF, van Rumste MM, Farquhar CM, Johnson NP, Mol BWM, Herbison P. Measuring outcomes in fertility trials: can we rely on clinical pregnancy rates?Fertil Steril 2010:94:1647-51. doi:10.1016/j. fertnstert 2009.11.018pmid:20056216.

$20 \mathrm{Kim}$ D, Child T, Farquhar C. Intrauterine insemination: a UK survey on the adherence to NICE clinical guidelines by fertility clinics. BMJ Open 2015;5:e007588. doi:10.1136/ bmjopen-2015-007588pmid:25979869.

Published by the BMJ Publishing Group Limited. For permission to use (where not already granted under a licence) please go to http://group.bmj.com/group/rights-licensing/ permissions 


\section{Key messages}

Most treatments offered by UK fertility treatment centres are not supported by good evidence

NICE provides clear advice on only 13 out 38 identified interventions, and systematic reviews were available for only 27

Information on harms is often poorly reported

Couples need clear advice on live birth rates and potential harms to make informed decisions

\section{Table}

\begin{tabular}{|c|c|c|c|}
\hline & $\begin{array}{l}\text { No of trials } \\
\text { (participants) in } \\
\text { review }\end{array}$ & $\begin{array}{l}\text { Effect on live birth rate: odds ratio } \\
(95 \% \mathrm{Cl})\end{array}$ & Quality of evidence \\
\hline Blastocyst culture $^{7}$ & $13(1630)$ & $\begin{array}{l}1.48(1.20 \text { to } 1.82) \text { compared with culture } \\
\text { to day } 2-3\end{array}$ & $\begin{array}{l}\text { High dropout rates and poor randomisation method descriptions for many } \\
\text { trials meant the evidence was judged low quality. Differences in live birth } \\
\text { rates were no longer significant ( } 1.3895 \% \mathrm{Cl} 0.96 \text { to } 1.99) \text { after low quality } \\
\text { studies were removed. Variable embryo transfer policies between groups }\end{array}$ \\
\hline $\begin{array}{l}\text { Endometrial } \\
\text { scratching }^{8}\end{array}$ & $9(1496)$ & $\begin{array}{l}\text { Relative risk } 1.42(1.08 \text { to } 1.85) \\
\text { compared with no injury }\end{array}$ & $\begin{array}{l}\text { Evidence quality was moderate because of low participant numbers and } \\
\text { limitations in the methods (in } 5 \text { studies both groups possibly received } \\
\text { some unintentional endometrial injury). Removal of the low quality studies } \\
\text { did not alter the effect estimate }\end{array}$ \\
\hline $\begin{array}{l}\text { Adherence } \\
\text { compounds }^{9}\end{array}$ & $6(1950)$ & $\begin{array}{l}1.41 \text { ( } 1.17 \text { to } 1.69) \text { compared with low } \\
\text { or no hyaluronic acid }\end{array}$ & $\begin{array}{l}\text { In the three studies ( } \mathrm{n}=324) \text { that only assessed high versus no hyaluronic } \\
\text { acid there was no significant effect (OR=1.35, } 95 \% \mathrm{Cl} 0.86 \text { to } 2.12) \text {. } \\
\text { Moreover, in some of the studies, multiple pregnancy rates were increased } \\
\text { because more embryos were transferred per woman in the intervention } \\
\text { groups }\end{array}$ \\
\hline Oral antioxidants ${ }^{10}$ & 4 small trials (277) & 4.21(2.08 to 8.51$)$ & $\begin{array}{l}\text { Only } 44 \text { live births recorded. One study had inadequate methods (the } \\
\text { numbers of participants initially randomised to each group were not } \\
\text { available) and a high unexplained dropout rate ( } 26 \%) \text {; in another, the } \\
\text { principal investigator had a commercial agreement with the manufacturer }\end{array}$ \\
\hline $\begin{array}{l}\text { Intrauterine } \\
\text { insemination in a } \\
\text { natural cycle }\end{array}$ & 14 (1867) & $\begin{array}{l}1.95(1.10 \text { to } 3.44) \text { in one trial }(n=396) \\
\text { compared with intercourse or expectant } \\
\text { management in a stimulated cycle }\end{array}$ & $\begin{array}{l}\text { For three other comparisons in the same review there was no significant } \\
\text { effect on live birth rates }\end{array}$ \\
\hline
\end{tabular}




\section{Figure}

\begin{tabular}{|c|c|}
\hline & $\begin{array}{l}13 \text { interventions had clear recommendations: } \\
11 \text { interventions recommended with specific populations: ovarian reserve test/AMH and antral follicle count; unstimulated cycle IUI; frozen } \\
\text { embryo transfer; surgical sperm retrieval; egg/embryo freezing; ICSI; vitrification of eggs/embryos; sperm freezing; ovulation induction and }\end{array}$ \\
\hline \multirow{3}{*}{$\begin{array}{l}\text { For } 38 \\
\text { interventions } \\
\text { we looked in } \\
\text { NICE CG156 for } \\
\text { recommendations }\end{array}$} & $\begin{array}{l}\text { cycle monitoring, sperm DNA test; thyroid antibodies } \\
2 \text { interventions not recommended: hysteroscopy, assisted hatching }\end{array}$ \\
\hline & $\begin{array}{l}19 \text { interventions were not mentioned or recommendations were unclear: SpermSlow, blastocyst culture, time lapse embryo imaging, IMSI, endometrial } \\
\text { scratching, adherence compounds, endometrial receptivity array, early embryo viability assessment, ovarian tissue freezing, artificial oocyte } \\
\text { activation, AneVivo, segmented IVF, oxidative stress levels in semen, dummy embryo transfer; PGS V1, PGS V2, PGD and PGD-A; stimulated cycle IUI }\end{array}$ \\
\hline & $\begin{array}{l}6 \text { interventions had recommendations for research: adjuvant luteal phase support treatments in IVF further research is needed to assess } \\
\text { efficacy of adjuvant luteal phase support treatments such as low dose aspirin, heparin, prednisolone, immunoglobulins and/or fat emulsions } \\
\text { (this also includes intralipid, cytokine testing, autoimmunity to HCG receptor, and quad therapy) }\end{array}$ \\
\hline $\begin{array}{l}\text { For } 38 \\
\text { interventions } \\
\text { we searched } \\
\text { PubMed for highest } \\
\text { level of evidence }\end{array}$ & $\begin{array}{l}27 \text { interventions: systematic review evidence } \\
18 \text { Cochrane systematic reviews: hysteroscopy, cytokine testing and treatment, aspirin, IUI in natural or stimulated cycles, surgical sperm } \\
\text { retrieval, SpermSlow, assisted hatching, blastocyst, ICSI, PGS-V1, vitrification of eggs/embryos, ovulation induction and cycle monitoring, } \\
\text { time lapse embryo imaging, IMSI, endometrial scratching, adherence compunds, oral antioxidant treatment, modified natural cycle IVF } \\
9 \text { non-Cochrane systematic reviews: artificial oocyte activation, egg/embryo freezing, Embryogen, frozen embryo transfer, ovarian reserve test, } \\
\text { PGS-v, PGD-A, spem DNA test, thyroid antibodies }\end{array}$ \\
\hline \multirow{3}{*}{$\begin{array}{l}\text { For } 11 \\
\text { interventions } \\
\text { with no systematic } \\
\text { review evidence, } \\
\text { we had to search } \\
\text { for lower level } \\
\text { of evidence }\end{array}$} & 8 interventions: single RCT or observational evidence \\
\hline & $\begin{array}{l}2 \text { RCTs: intralipid infusion; sperm freezing } \\
6 \text { observational studies: ovarian tissue freezing, endometrial receptivity array, early embryo viability assessment, AneVivo, PGD; autoimmunity } \\
\text { to HCG receptor }\end{array}$ \\
\hline & $\begin{array}{l}3 \text { interventions: expert opinion or mechanism } \\
\text { Segmented IVF, dummy embryo transfer, and quad therapy }\end{array}$ \\
\hline
\end{tabular}

AMH=anti-mullerian hormone; IUI=Intrauterine insemination; ICSI=Intracytoplasmic sperm injection; IMSI=Intracytoplasmic morphologically selected sperm injection; PGS=Pre-genetic screening; $\mathrm{PGD}=$ Preimplantation genetic diagnosis

\section{Evidence search for 38 interventions offered by UK fertility clinics}

